\title{
Simultaneous Determination of C22-C26 Very Long - Chain Fatty Acids Following tert-Butyldimethylsilyl Derivatization by Stable Isotope GC- MS for the Screening of Adrenoleucodystrophy
}

\author{
Hye-Ran Yoon \\ Ssangmun-dong 419, Dobong-gu, Dept. of Biomedical \& Clinical Analytical Chemistry, \\ College of Pharmacy, Duksung Women's University, Seoul 132-714, South Korea
}

(Received August 14, 2007; Accepted August 30, 2007)

\begin{abstract}
A rapid analytical method was developed to quantify very long-chain fatty acids (VLCFAs, C22:0, C24:0, C26:0) in human plasma with good sensitivity and specificity using tert-butyldimethylsilyl (TBDMS) derivatization and stable isotope GC-MS selective ion monitoring (GC-MS/SIM). Two-hundred and fifty $\mu \mathrm{L}$ of plasma was fortified with deuterated stable isotope internal standards (d3-C22:0, d3-C24:0, d3-C26:0) and standard mixtures of chloroform and methanol, and then extracted with hexane and acetonitrile. To upper layer of liquid-liquid-extraction, N-(t-Butyldimethylsilyl)-N-methyltrifluoroacetamide was added and then heated to 60 ${ }^{\circ} \mathrm{C}$ for $30 \mathrm{~min}$ to produce the TBDMS derivatives. Derivatives of VLCFAs were analyzed by GC-MS/SIM. Calibration curves showed a linear relationship for the target compounds in the concentration range of $10^{-4} \sim 2 \times$ $10^{3} \mu \mathrm{g} / \mathrm{mL}$ with the correlation coefficient ranging from 0.996 to 0.999 . The limit of quantification for the plasma was $10^{-4} \sim 2 \times 10^{-4} \mu \mathrm{g} / \mathrm{mL}(\mathrm{S} / \mathrm{N}=3$ ). When applied to the plasma specimens of patients with peroxisomal disorder, X-linked adrenoleucodystropy (ALD, Mckusick 202370), the method clearly differentiated normal subjects from ALD patients. The $\mathrm{C} 24: 0 / \mathrm{C} 22: 0$ and $\mathrm{C} 26: 0 / \mathrm{C} 22: 0$ ratios were significantly elevated in the plasma of patients with $\mathrm{X}$-linked ALD compared to normal subjects. The new developed method might be useful for a rapid and sensitive diagnosis of X-linked ALD and other peroxisomal disorders.
\end{abstract}

Keywords $\square$ GC-MS/SIM, TBDMS derivative, docosanoic, tetracosanoic, hexacosanoic, peroxisomal disorder, X-linked Adrenoleucodystropy

\section{INTRODUCTION}

The peroxisome is a cellular organelle with diverse metabolic function, usually contains one or more enzymes. A major role of peroxisome is break down the peroxisomal fatty acid molecules by oxidation process. The enzyme deficiencies are related to the accumulation of very long-chain fatty acids (VLCFAs) (with 22 or more carbon atoms) in tissues and body fluids (Harris et al., 1990, Martinez et al., 1992, Hommes et al., 1991). The VLCFAs accumulation can be used as a marker for most peroxisomal disorders, especially X-linked adrenoleukodystrophy (X-ALD). ALD is one of a group of rare, genetic disorders called the leukodystrophies that cause damage to the myelin sheath, an insulating membrane that surrounds nerve

*Corresponding author

Tel: +82-2-901-8387, Fax: +82-2-997-1990

E-mail: hyeran11@duksung.ac.kr cells in the brain (Rizzo et al., 1989, Kaplan et al., 1993). Patients with ALD accumulate high levels of saturated, VLCFAs in the brain and adrenal cortex because they do not produce the enzyme that breaks down these fatty acids in the normal manner. Diagnosis of X-ALD is mainly based on clinical features (abnormal withdrawal or aggression, poor memory, and poor school performance ) and biochemical abnormality of elevation of the plasma concentration of hexacosanoic acid (C26:0) as well as the ratios of hexacosanoic, and lignoceric acid (C24:0), relative to behenic acid (C22:0), which are biochemical markers for X-ALD. Usually their concentration of tissues and body fluids in ALD patients are higher than those of normal subjects (Aveldano et al., 1996, Moser et al., 1983 and 1987). Therefore, measurement of VLCFAs in body fluid function is one of the most important diagnostic criteria for the demonstration of an abnormal function of peroxisome (Martinez et al., 1992, Caruso et al., 1991). There are reports on the analysis of FAs by chromatographic methods, such as LC, GC, 
GC-MS (Onkenhout et al., 2005). Gas chromatography-mass spectrometry (GC-MS) is a technique widely used for the identification of FAs in biological mixtures, usually as their methyl, benzyl and trimethylsilyl (TMS) esters (Onkenhout et al., 1989, Hintze et al., 1973, Kuksis et al., 1976, Welz et al., 1990, Caruso et al., 1991). These derivatives, however, are not considered satisfactory for unequivocal identification or sensitive detection purposes. The mass spectra obtained are typically dominated by intense ions at the lower mass, characteristic of the ester moiety rather than the structure of the acid itself, and the abundance of the high mass such as molecular ions, decreases with increasing the chain length of the acids.

Methyl derivatives do not differentiate it from endogenous methylester found in the body. Silyl-derivatives easily break down when exposed to moisture. Trimethylsilyl derivatives of fatty acids do not always give quantitative yield. These disadvantages can be somewhat overcome the moisture sensitivity of silyl-derivatives and allows the quantitative derivatization by using tert-butyldimethylsilyl (TBDMS) derivatives.

This study was undertaken to investigate clinical application of the developed method and its practical use. We report a sensitive, efficient and rapid analytical method for the determination of VLCFAs following TBDMS derivatization by stable isotope GC- MS for diagnosis and follow-up monitoring of inherited metabolic disorders, X-ALD.

\section{MATERIALS AND METHODS}

\section{Materials}

Very long-chain fatty acids standards [docosanoic (behenic, C22:0), tetracosanoic (lignoceric, C24:0), and hexacosanoic (C26:0) acids]] were purchased from Sigma-Aldrich (MA, USA). Deuterium labeled stable isotope (d4-C22:0, d4-C24:0, and d4-C26:0) as an internal standards (I.S.) were purchased from Cambridge Isotope Laboratories, Inc (MA, USA). Acetonitrile, hexane, chloroform, methanol and all chemicals and solvents for GC-MS analyses were of analytical-reagent grade and purchased from Sigma-Aldrich (MA, USA) or Merck (Darmstadt, Germany). $N$-Methyl- $N$-( $t$-butyldimethylsilyl) trifluoroacetamide(MTBSTFA) as silylating reagent was purchased from Sigma-Aldrich (MA, USA).

Distilled water was prepared by Millipore-Milli Q ${ }^{\mathrm{TM}}$ (Tokyo, Japan). Thermo vap (Pierce model 18780, USA) was used for evaporation and derivatisation. A shaker (TAITEC, Tokyo, Japan) was used for both mixing specimen and derivative reactions.

\section{Preparation of standards solution}

Stock solutions of each docosanoic (C22:0), tetracosanoic (C24:0), and hexacosanoic (C26:0) acids studied were prepared by dissolving $10 \mathrm{mg}$ of target compound in $10 \mathrm{~mL}$ of chloroform $\left(\mathrm{CHCl}_{3}\right)$-methanol $\left(\mathrm{CH}_{3} \mathrm{OH}\right)(2: 1, \mathrm{v} / \mathrm{v})$ mixture (final $1,000 \mu \mathrm{g} / \mathrm{mL}$ ). These stock solutions were further diluted with the same organic solvent to prepare working solutions of varying concentrations from 10 to $100 \mu \mathrm{g} / \mathrm{mL}$. The internal standard solution was dissolved at a concentration of $1 \mathrm{mg} / \mathrm{mL}$ in chloroform - methanol $(2: 1, \mathrm{v} / \mathrm{v})$ mixture.

\section{Subjects and sample collection}

Plasma specimens were obtained from healthy volunteers (618 years of age) without remarkable medical histories and three patients (8-19 years of age) with confirmed X-ALD by clinical presentation, MRI findings and biochemical assay. All patient and control subjects in this study underwent the same diagnostic procedure in the same facilities. Patient groups received no treatment before plasma samples were obtained. The plasma specimens were collected in polyethylene bottles and immediately stored at $-20^{\circ} \mathrm{C}$ until analysis.

\section{Gas chromatography - mass spectrometry}

Analyses were carried out on a Hewlett-Packard 6890 Series II GC-MS system (PA, USA) consisting of a Model 6890 gas chromatograph, a Model 5973 mass-selective detector, a HP Hewlett-Packard 3365 MSD Chemstation, and a Model 6890 series injector. For separation of VLCFAs, a HP Ultra-2 (25 m $\times 0.25 \mathrm{~mm}$ I.D., cross-linked 5\% phenyl-methyl silicone, 0.25 $u \mathrm{~m} \mathrm{~d}_{\mathrm{f}}$ film thickness) fused-silica capillary column was used. The column head pressure of helium as a carrier gas was set to $130 \mathrm{kPa}$ (flow-rate of $0.8 \mathrm{~mL} / \mathrm{min}$ ), and split ratio was 1:10. The GC oven temperature was programmed from $200^{\circ} \mathrm{C}(5 \mathrm{~min}$ hold) to $300^{\circ} \mathrm{C}$ at a rate of $8^{\circ} \mathrm{C} / \mathrm{min}(5 \mathrm{~min}$ hold $)$. The temperatures of the interface and injector were $300^{\circ} \mathrm{C}$ and $280 \mathrm{C}$, respectively. The mass spectrometer was operated at $70 \mathrm{eV}$ in the electron impact (EI) mode, and the SIM mode was used for quantifying VLCFAs in human plasma. A dwell time of 100 $\mathrm{ms}$ and a relative EM voltage of $400 \mathrm{~V}$ higher than that in the scanning mode were chosen for each ion monitored.

Two $\mu \mathrm{L}$ of the final derivatized aliquots was injected into the GC-MSD. The mass fragment and the chromatogram of the derivatives were confirmed by obtaining the data in full scan mode with a scan range from $\mathrm{m} / \mathrm{z} 50$ to 550 (Fig. 1). Table I shows the ions used for quantification (QI) and confirmation ions (CI). 


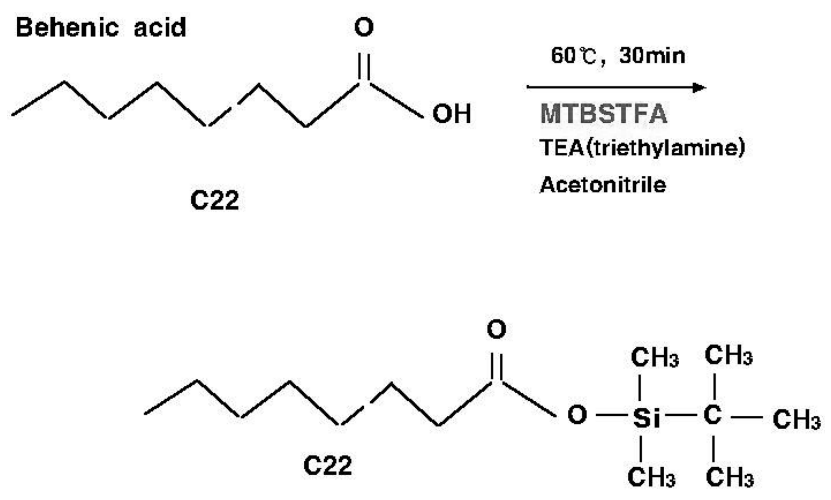

Fig. 1. Reaction scheme for TBDMS derivatives of very longchain fatty acids.

Table I. Comparison of TBDMS and methyl derivatization regarding major parameters of selected ions for analyses of VLCFAs

\begin{tabular}{lcccccc}
\hline \multicolumn{1}{c}{ Compounds } & \multicolumn{6}{c}{ methyl derivative } \\
& F.W. & M + & RT & RRT & QI & CI \\
\hline behenic acid (C22:0) & 340 & 354 & 13.7 & 1.003 & 354.6 & 311.5 \\
lignoceric acid (C24:0) & 368 & 382 & 15.7 & 1.002 & 382.5 & 339.6 \\
cerotic acid (C26:0) & 396 & 410 & 17.5 & 1.001 & 410.6 & 367.6 \\
\hline \multicolumn{6}{c}{ TBDMS derivative } \\
\hline behenic acid (C22:0) & 340 & 354 & 13.7 & 1.002 & 397.4 & 439.5 \\
lignoceric acid (C24:0) & 368 & 382 & 15.7 & 1.002 & 425.5 & 467.5 \\
cerotic acid (C26:0) & 396 & 410 & 17.5 & 1.003 & 453.5 & 495.6 \\
\hline
\end{tabular}

\section{Sample preparation and derivatisation}

For the extraction and methylation of VLCFAs in plasma samples, a previously reported method (Paik et al., 1999) was modified. To a $250 \mu \mathrm{L}$ aliquot of plasma was added $50 \mu \mathrm{L}$ (10 $\mu \mathrm{g} / \mathrm{mL}$ ) of I.S. mixture (d4-C22:0, d4-C24:0, and d4-C26:0) and cooled for $1.5 \mathrm{~h}$ in the refrigerater.

The method of Lepage and Roy (Lepage et al., 1984) modified by Cohen et al. (Cohen et al., 1988) was used using deuterium labeled VLCFAs as an internal standards. For methyl derivatives, to a plasma with I.S. mixture was added $1 \mathrm{~mL}$ of methanol and methylene chloride (3:1, v/v) mixture solution, a $200 \mu \mathrm{L}$ of acetyl chloride and potassium carbonate. The mixture was vortexed for $2 \mathrm{~min}$ at room temperature. Then VLCFAs were transferred to the methylene chloride phase by centrifugation for $3 \mathrm{~min}$ at $3000 \mathrm{rpm}$. After cooling with ice bath, $4 \mathrm{~mL}$ of hexane was added. The hexane phase was transferred into another glass vial and $2 \mathrm{~mL}$ of acetonitrile was added. The supernatant hexane phase obtained by centrifugation at 3,000 rpm for $3 \mathrm{~min}$ was evaporated to dryness in a vacuum evaporator under a gentle stream of nitrogen. The dry residue

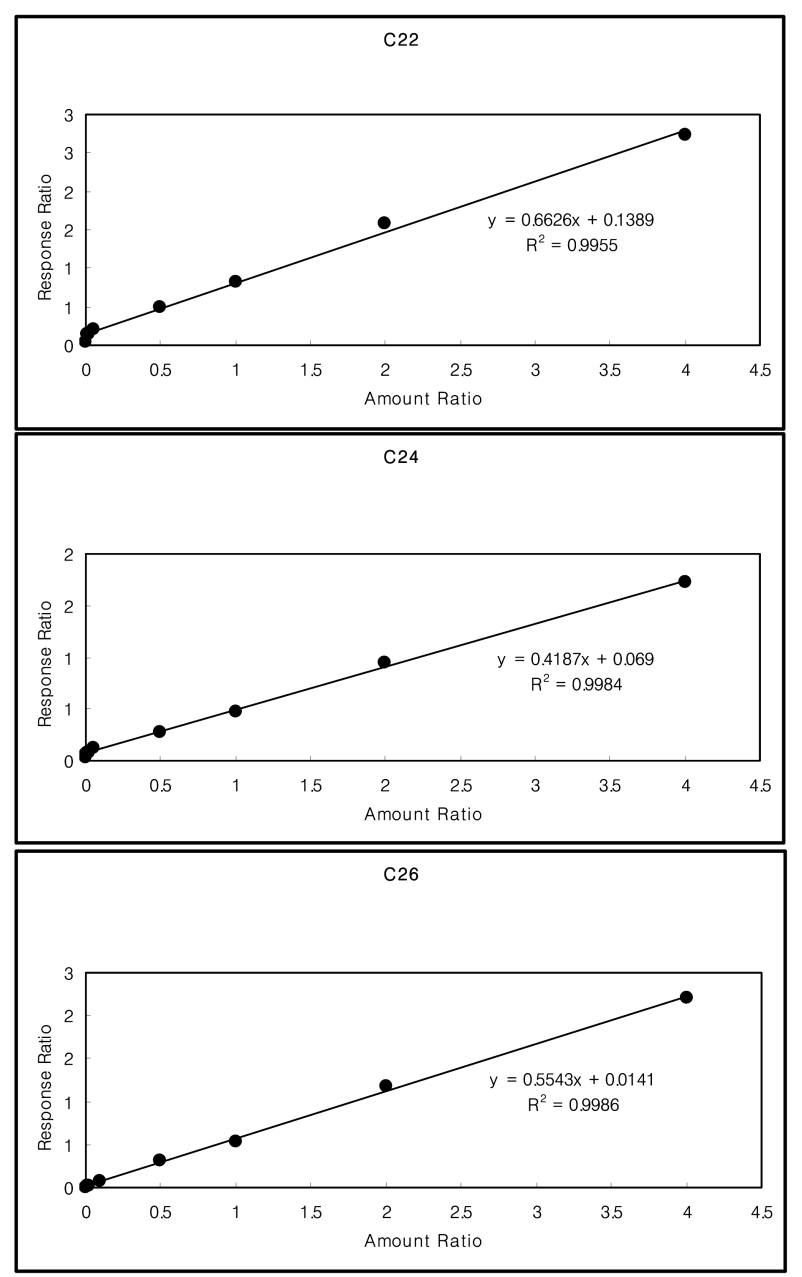

Fig. 2. Linear dynamic range of VLCFAs under GC/MS-SIM analyses.

was dissolved with $50 \mu \mathrm{L}$ of hexane.

For silyl (TBDMS) derivatives, the supernatant hexane phase from the above procedure was dried in a vacuum evaporator under a gentle stream of nitrogen. To the residue, Tert-butyldimethylsilyl(TBDMS) derivatives of VLCFAs (C22:0, C24:0, and C26:0) was performed by adding $50 \mu \mathrm{L}$ of methyl tert-butyldimethylsilyl trifluoroacetamide (MTBSTFA), triethylamine and acetonitrile and then reacted at $60^{\circ} \mathrm{C}$ for $30 \mathrm{~min}$. The completed TBDMS derivatives were cooled for $5 \mathrm{~min}$ and transferred to a GC auto-sampler vial and directly analyzed by GC-MS.

The standard samples containing 0.1-1.0 ng each of the VLCFAs was prepared to determine detection limits. Determination of the signal-to-noise $(\mathrm{S} / \mathrm{N}=3)$ ratio is performed by comparing measured signals from samples with known low concentrations of analyte with those of water blank as their 
TBDMS derivatives.

\section{Calibration}

Calibration curves of each VLCFAs were set up over a concentration range of $0.05-100.0 \mu \mathrm{g} / \mathrm{mL}$ and are shown in Fig. 2. For the standard curve of C22:0, C24:0, and C26:0, 0, 5, 25, 50 , and $100 \mu \mathrm{L}$ of the working mixture solution $(100 \mu \mathrm{g} / \mathrm{mL})$ and $50 \mu \mathrm{L}(10 \mu \mathrm{g} / \mathrm{mL})$ of internal standards (d4-C22:0, d4C24:0, and d4-C26:0) was added to $250 \mu \mathrm{L}$ of blank plasma. The concentrations of $\mathrm{C} 22: 0, \mathrm{C} 24: 0$, and $\mathrm{C} 26: 0$ ranged from 0.5 to $10.0 \mu \mathrm{g} / \mathrm{mL}$. After the addition of $0.5 \mu \mathrm{g}$ of the internal standard, each sample was extracted and derivatized according to the method as described above for the plasma sample.

For the quantitative analyses, relative peak area ratios of VLCFAs standard over internal standard were calculated and plotted in the calibration range of each VLCFAs.

\section{RESULTS AND DISCUSSION}

Fig. 1 shows one-step TBDMS derivatisation reaction scheme of very long- chain fatty acids, e.g., behenic acid. As a result of reaction the carboxylic functional group of behenic acid was silylated to TBDMS ester with MTBSTFA, triethylamine in acetonitrile at $60 \mathrm{C}$ for $30 \mathrm{~min}$. To find the optimal reaction time of TBDMS derivatives for VLCFAs, the reaction was performed for various time $(10,20,30,40,50$, and $60 \mathrm{~min})$ at $60^{\circ} \mathrm{C}$. In this study, the reaction time for $30 \mathrm{~min}$ at $60^{\circ} \mathrm{C}$ showed the highest reaction efficiency and least interference peak.

It is necessary to use a technique comprising both highly selective and sensitive in order to determine the low concentration of VLCFAs accurately. However, previously used methylation method for their determination has often been unsatisfactory when used for fast, sensitive identification purposes, e.g., when used to determine the abundance of high mass, the formation of an intense molecular ion and a GC property. Moreover, the method is weak to the moisture (Caruso et al., 1991). The present derivatization with TBDMS was completed within 30 $\min$ at $60^{\circ} \mathrm{C}$. All the carboxyl groups of VLCFAs were readily converted to their corresponding TBDMS esters. In preliminary work, TBDMS-VLCFAs resulted in an amendable work-up process and, when stored in a derivatizing reagent, were more stable than TMS derivatives. The TBDMS derivatives have longer retention times than methyl one but, demonstrate good GC properties with rich abundance of high mass ions, that are suitable for the selected ion monitoring.

Table I presents information on VLCFAs for selected ion monitoring mode including quantification and confirmation ions, formula weight $(\mathrm{FW})$, derivatized molecular peak $\left(\mathrm{M}^{+}\right)$, retention time (RT), and relative retention time (RRT). The RRT was calculated from the retention time of each VLCFAs versus each I.S. Quantification ion (QI) was chosen from the ion that has a $\mathrm{m} / \mathrm{z}>100$ on spectra and does not interfere with other compounds on chromatogram, mostly $[\mathrm{M}-57]^{+}$ion. Confirmation ion (CI) was chosen from the VLCFAs-TBDMS molecular ions even if it shows low spectral intensity on spectra (Table I). The identification of a compound was based on the presence of the co-elution of two selected ion species, QI and $\mathrm{CI}$, on the GC-MS chromatogram in plasma specimen. Two ions were chosen for quantification and confirmation in order to identify the target compounds from the possible co-eluting interference peak. The ratio of their peak intensity (quantification ion / confirmation ion) was another important parameter used for accurate confirmation.

When compared TBDMS derivative ion with methyl derivative ion as shown in Table I, much higher mass ions (mostly $>$ m/z 395) were chosen than methyl derivative. High mass ions were selected for the sake of protection from the possibility of interference in biological specimens (Table I).

In case of methyl derivative, most of the confirmation ions chosen were $[\mathrm{M}-15]^{+}$, which indicates the loss of a methyl radical from the derivatized molecular ion (Table I).

Quantification of VLCFAs was achieved by adding deuterated internal standards to a plasma sample and monitoring two quantification and confirmation ion on the GC-MS instrument from each of the VLCFAs and their quadrideuterated analogues by selected ion monitoring experiments. Quantitative determination was performed by calculating the relative peak area of the quantification ion of the VLCFAs versus internal standard. The ratios $\mathrm{C} 24: 0 / \mathrm{C} 22: 0$ and $\mathrm{C} 26: 0 / \mathrm{C} 22: 0$ were also determined in order to make sure the diagnosis.

A six-point standard curve was constructed after analyzing mixtures of the labeled and unlabelled VLCFAs over the range found for plasma specimens. Fig. 2 shows regression line with correlation coefficient of target VLCFAs-TBDMS derivatives. The correlation coefficients for each VLCFAs gave an $\mathrm{R}^{2}$ of better than 0.996 .

The calibration curves were linear in the concentration range of $10^{-4} \sim 2 \times 10^{3} \mu \mathrm{g} / \mathrm{mL}$ for VLCFAs on plasma specimen (Fig. 2 ). The minimum concentration of VLCFAs determined by this method was calculated by the method described by PerezBendito and Silva ( $3 \mathrm{~S}_{\mathrm{bi}}$-criterion) (Perez-Bendito et al., 1988). The limits of quantitation (LOQ) was found to be $10^{-4} \sim 2 \times 10^{-4}$ 
Table II. Linear dynamic range, LOD and LOQ of TBDMS derivatives of VLCFAs

\begin{tabular}{lcc}
\hline \multicolumn{1}{c}{$\begin{array}{c}\text { Compounds } \\
(\text { unit } ; \mu \mathrm{g} / \mathrm{mL})\end{array}$} & Linear range & LOQ(S/N=3) \\
\cline { 2 - 3 } behenic acid $(\mathrm{C} 22: 0)$ & $10^{-4} \sim 2 \times 10^{3}$ & methyl derivative \\
lignoceric acid $(\mathrm{C} 24: 0)$ & $10^{-4} \sim 2 \times 10^{3}$ & $10^{-2}$ \\
cerotic acid $(\mathrm{C} 26: 0)$ & $10^{-4} \sim 2 \times 10^{3}$ & $10^{-3}$ \\
\hline & & TBDMS derivative \\
\cline { 2 - 3 } behenic acid (C22:0) & $10^{-4} \sim 2 \times 10^{3}$ & $10^{-4} \sim 2 \times 10^{-4}$ \\
lignoceric acid $(\mathrm{C} 24: 0)$ & $10^{-4} \sim 2 \times 10^{3}$ & $10^{-4} \sim 2 \times 10^{-4}$ \\
cerotic acid $(\mathrm{C} 26: 0)$ & $10^{-4} \sim 2 \times 10^{3}$ & $10^{-4} \sim 2 \times 10^{-4}$ \\
\hline
\end{tabular}

Table III. Quantification of behenic, lignoceric and cerotic acid in plasmas

\begin{tabular}{|c|c|c|c|c|c|}
\hline \multirow{2}{*}{$\begin{array}{l}\text { Reference value } \\
\text { ( unit ; mM ) }\end{array}$} & $\mathrm{C} 22: 0$ & C24:0 & C26:0 & C24:0/C22: & $26: 0 / \mathrm{C} 22: 0$ \\
\hline & $\leq 96$ & $\leq 91.4$ & $\leq 1.31$ & $\leq 1.39$ & $\leq 0.021$ \\
\hline patient 1(ALD) & 36.16 & 66.59 & 2.60 & 1.84 & 0.0720 \\
\hline patient $2(\mathrm{ALD})$ & 22.87 & 37.54 & 1.34 & 1.64 & 0.0584 \\
\hline patient $3(\mathrm{ALD})$ & 21.51 & 45.35 & 2.67 & 2.11 & 0.1243 \\
\hline healthy subject 1 & 25.41 & 26.44 & 0.19 & 1.04 & 0.0075 \\
\hline healthy subject 2 & 35.68 & 30.31 & 0.05 & 0.85 & 0.0013 \\
\hline healthy subject 3 & 54.51 & 49.81 & 0.18 & 0.91 & 0.0032 \\
\hline pooled plasma 1 & 18.35 & 16.49 & 0.00 & 0.00 & 0.0000 \\
\hline pooled plasma 2 & 14.13 & 12.70 & 0.00 & 0.00 & 0.0000 \\
\hline pooled plasma 3 & 20.96 & 19.57 & 0.00 & 0.00 & 0.0000 \\
\hline
\end{tabular}

$\mu \mathrm{g} / \mathrm{mL}$ ( $\mathrm{S} / \mathrm{N}=3$ ) (Table II). TBDMS derivative was more than 10 times superior to methyl derivative in terms of sensitivity.

No interference peak was in the quantification of standards VLCFAs extracted from plasma after VLCFAs fortification (Fig. 3). There were overlapping peaks for VLCFAs on the

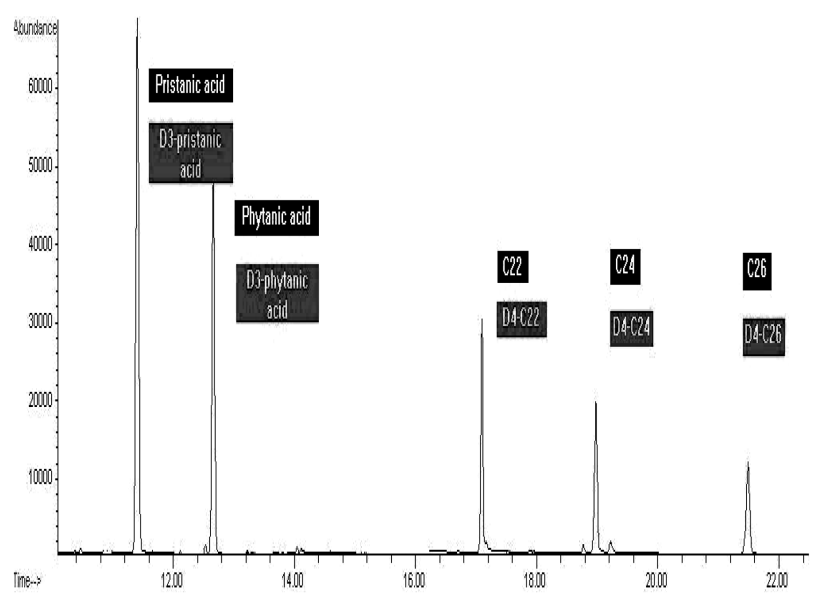

Fig. 3. Standards VLCFAs profiles following TBDMS derivatives of docosanoic(C22:0), tetracosanoic(C24:0), and hexacosanoic(C26:0) on GC-MS-SIM chromatogram.

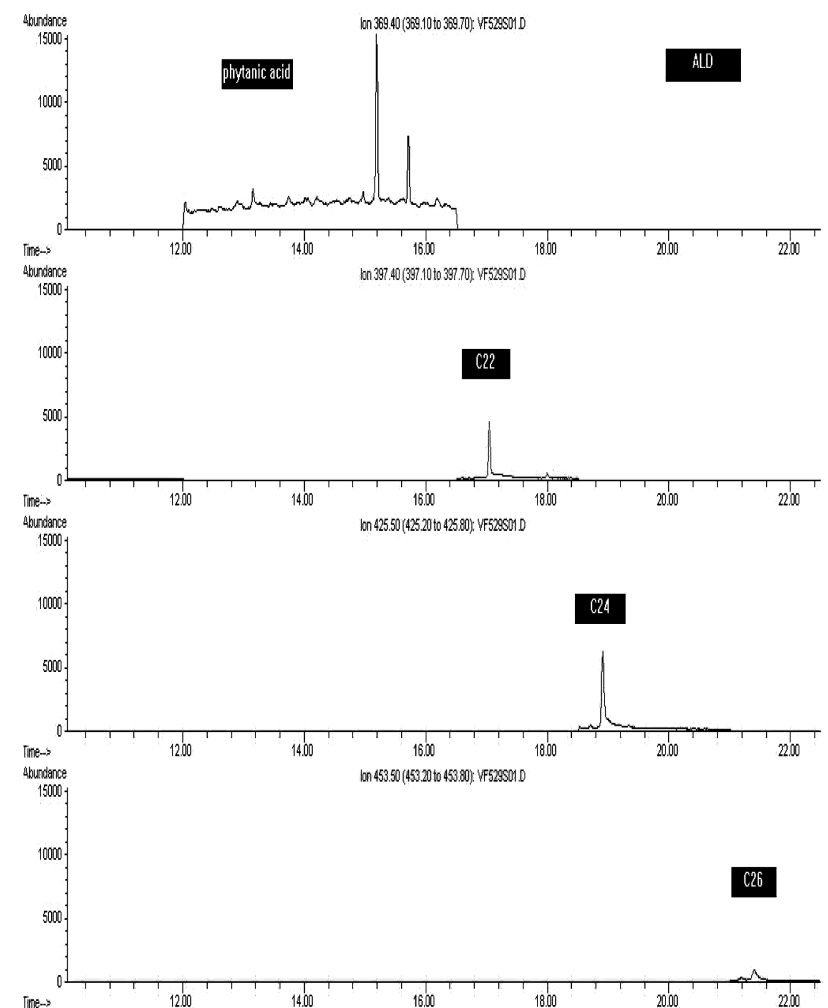

Fig. 4. Plasma VLCFAs profiles following TBDMS derivatives of docosanoic(C22:0), tetracosanoic(C24:0), and hexacosanoic (C26:0) on GC-MS-SIM chromatogram from healthy control.

chromatogram. Each compound was completely separated on GC-MS selected ion monitoring mode. The result of quantification showed an unequivocal separation and discrimination between healthy control subjects and patient with X-ALD. SIM chromatograms of C22:0, C24:0 and C26:0 VLCFAs in plasma were showed in Fig. 4 (healthy control subjects who was volunteer and ruled out through basic health promotion testing) and Fig. 5 (patient with X-ALD), respectively.

Table III lists VLCFAs C24:0, C26:0 and ratio of C24:0 / $\mathrm{C} 22: 0, \mathrm{C} 26: 0 / \mathrm{C} 22: 0$ as diagnostic markers for the diagnosis of X-ALD. Relatively increased concentration of $\mathrm{C} 26: 0$ and the ratios of $\mathrm{C} 26: 0, \mathrm{C} 24: 0$ to $\mathrm{C} 22: 0$ from plasma of X-ALD patients was observed in comparison with healthy control samples (Table III).

Individul normal healthy plasma proved by health promotion testing was pooled in single large test tube and mixed well for homogenous plasma mixture. The expiration date for pooled plasma ranges up to 1 year, if kept refrigerated under $-20^{\circ} \mathrm{C}$. C26:0, most critical marker for the diagnosis of ALD was not detected in pooled plasmas and was within normal range in healthy subjects whereas enormously elevated in plasma with 


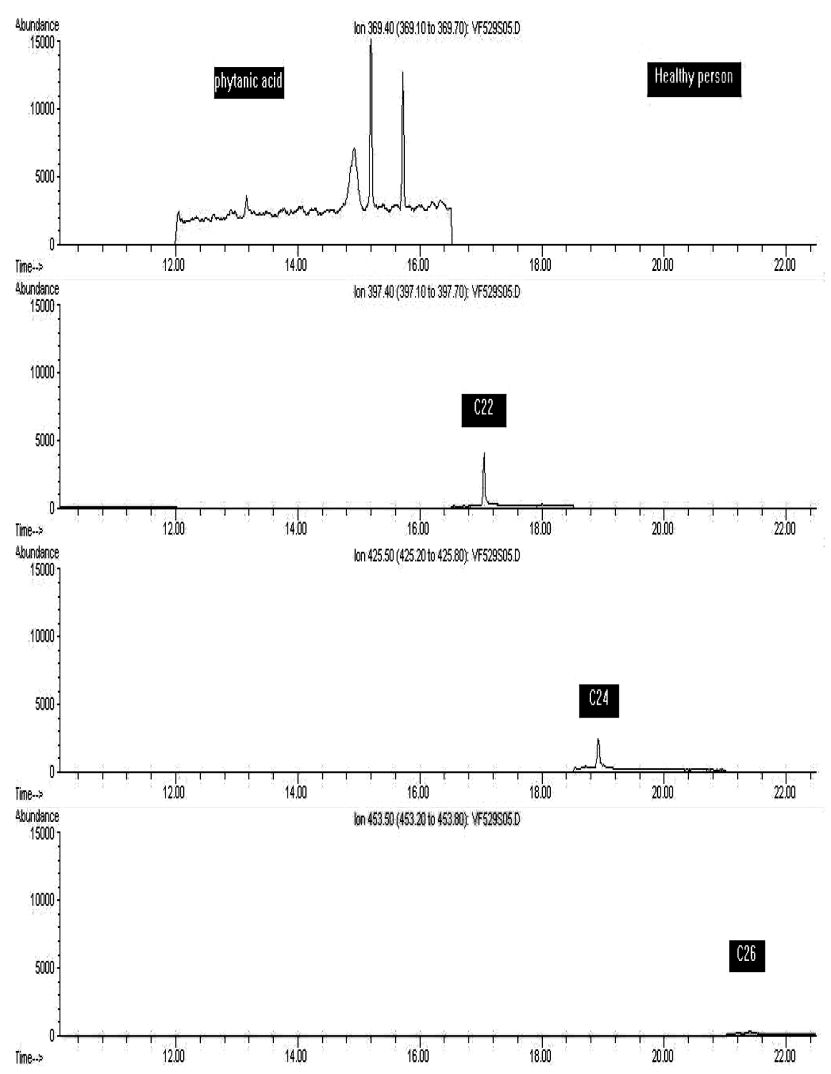

Fig. 5. Plasma VLCFAs profiles following TBDMS derivatives of docosanoic(C22:0), tetracosanoic(C24:0), and hexacosanoic (C26:0) on GC-MS-SIM chromatogram from ALD patients.

ALD patient 1, 2 and 3. The ratios of $\mathrm{C} 26: 0 / \mathrm{C} 22: 0$ and $\mathrm{C} 24: 0 /$ C22:0 obtained from the childrens' plasma of both healthy control group and X-ALD patients were consistent with those obtained by Jakobs and colleagues (Caruso et al., 1993). The ratio of $\mathrm{C} 24: 0 / \mathrm{C} 22: 0$ does not differentiate X-ALD patients from healthy control group meaning that the parameter is just for reference. The ratio of $\mathrm{C} 26: 0 / \mathrm{C} 22: 0$ was between $33 \%$ and $233 \%$ higher in plasma with X-ALD patients than in control plasma.

Major advantage of the proposed method was VLCFAsTBDMS derivatives featuring that characteristic $[\mathrm{M}-57]^{+}$high mass ions permitted highly selective detection of trace $\mathrm{C} 26: 0$ VLCFAs even though in the presence of higher level of coextracted endogenous plasma interferences. In addition, TBDMS derivative is more stable than methyl derivative when exposed to moisture.

A simple, rapid, and resistant to contaminants and inexpensive method for the determination of VLCFAs in plasma was developed by GC-MS. The carboxyl group of VLCFAs was silylated to TBDMS esters. In the present method, VLCFAs analysis was satisfactorily performed without interfering with the substance and the GC-MS system proved appropriate for trace level determination of these compounds with TBDMS derivatives. Therefore, the described method can be used for routine analysis of VLCFAs in the diagnosis of X-ALD and many peroxisomal disorders in plasma samples for clinical application. This method also may be used the monitoring of peroxisomal disorder patients on dietary therapy.

\section{ACKNOWLEDGEMENTS}

This study was supported by a research grant 2006 from the Duksung Women's University, Seoul, Korea. I am indebted to Mr. Seungwoo Kang (Biocore, co.) for his skillful technical assistance and critical comments for this experiment.

\section{REFERENCES}

Bligh, E.G., Dyer, W.J. (1959). A rapid method of total lipid extraction and purification. Can. J. Biochem. Physiol. 37, 911918.

Caruso, U., Fowler, B., Erceg, M., Romano, C. (1991). Determination of very-long-chain fatty acids in plasma by a simplified gas chromatographic-mass spectrometric procedure. J Chromatogr. 2, 562(1-2), 147-52.

Cohen, Z., Vonshak, A., Richmond, A. (1988). Effect of environmental conditions on fatty acid composition of the red alga Porphyridium cruentum: Correlation to growth rate. J. Phycol. 24, 328- 332.

Jakobs, C., van den Heuvel, C.M., Stellaard, F., LargilliÃ̈re, C., Skovby, F., Christensen, E. (1993). Diagnosis of Zellweger syndrome by analysis of very long-chain fatty acids in stored blood spots collected at neonatal screening. $J$ Inherit Metab Dis. 16(1): 63-6.

Kang, J.X., Wang, J. (2005). A simplified method for analysis of polyunsaturated fatty acids. BMC Biochem. 24, 6:5.

Kuksis, A., Myher, J.J., Marai, L, Geher, K. (1976). Estimation of plasma free fatty acids as the trimethylsilyl (TMS) esters. Anal Biochem. 70(2), 302-12.

Lepage, G., Roy, C. (1984). Improved recovery of fatty acid through direct transesterification without prior extraction or purification. J. Lipid Res. 25, 1391-1396.

Mansour, M.P. (2005). Reversed-phase high-performance liquid chromatography purification of methyl esters of $\mathrm{C}(16)-\mathrm{C}(28)$ polyunsaturated fatty acids in microalgae, including octacosaoctaenoic acid [28:8(n-3)]. J Chromatogr A. 2, 1097(12):54-8

Mondello, L, Tranchida, P.Q., Dugo, P., Dugo, G. (2006). Rapid, micro-scale preparation and very fast gas chromatographic separation of cod liver oil fatty acid methyl esters. J Pharm Biomed Anal. 28, 41(5), 1566-70.

Moser, H. W., Moser, A. B. (1991) Techniques in Diagnostic Human Biochemical Genetics.: 177-191.

Onkenhout, W., van der Poel, P.F., van den Heuvel, M.P. (1989). Improved determination of very-long-chain fatty acids in 
plasma and cultured skin fibroblasts: applications to the diagnosis of peroxisomal disorders. J Chromatogr. 29, 494, 31-41.

Paik, M.J., Lee, K.O., Shin, H.S. (1999). Determination of verylong-chain fatty acids in serum by gas chromatographynitrogen-phosphorus detection following cyanomethylation. $J$ Chromatogr B. 8, 721(1), 3-11.
Perez-Bendito, D., Silva, M. (1988). Kinetic Methods in Analytical Chemistry; Ellis Horwood: Chichester, pp. 251-253.

Welz, W., Sattler, W., Leis, H.J., Malle, E. (1990). Rapid analysis of non-esterified fatty acids as methyl esters from different biological specimens by gas chromatography after one-step esterification. J Chromatogr. 6, 526(2, 319-29. 